\title{
HSP70 Expression in the Mouse Dental Pulp after Immediate Teeth Separation
}

\author{
Keisuke Nakano $^{1)}$, Shinnosuke Saito ${ }^{2)}$, Atsushi Nabeyama ${ }^{1)}$, Shintaro Oishi ${ }^{1,4)}$, Masahiro Sato ${ }^{4)}$, Yukiko Yokoi ${ }^{5)}$, \\ Naoto Osuga ${ }^{2,5)}$, Norimasa Okafuji ${ }^{3)}$ and Toshiyuki Kawakami ${ }^{1}$ \\ ${ }^{1)}$ Hard Tissue Pathology Unit, Matsumoto Dental University Graduate School of Oral Medicine, Shiojiri, Japan \\ ${ }^{2}$ Oral Health Analysis Unit, Matsumoto Dental University Graduate School of Oral Medicine, Shiojiri, Japan \\ ${ }^{3)}$ Clinical Evaluation Unit, Matsumoto Dental University Graduate School of Oral Medicine, Shiojiri, Japan \\ ${ }^{4}$ Department of Endodontics and Operative Dentistry, Matsumoto Dental University School of Dentistry, Shiojiri, Japan \\ ${ }^{5}$ Department of Pediatric Dentistry, Matsumoto Dental University School of Dentistry, Shiojiri, Japan \\ (Accepted for publication, August 22, 2012)

\begin{abstract}
We examined the change of HSP70 expression in the mouse dental pulp which was exposed to experimental teeth separation by immunohistochemistry as a model for conservative dental treatment. Eight week-old 18 male ddY mice were used and a wedge was inserted between upper $1^{\text {st }}$ and $2^{\text {nd }}$ molars for 3 hours. In the experimental group, HSP70 expression increased in the dental pulp tissues. The expression was the greatest at 24 hours after which it gradually decreased to become similar to the level of the control group at 1 no severe damage occurs upon clinical application of a wedge insertion.
\end{abstract} \\ week. These results suggest that when immunohistochemical expression of HSP70 is used as an index, almost
}

Key words: HSP70, Immediate teeth separation, Dental Pulp, Immunohistochemistry, Mechanical stress

\section{Introduction}

In the dental pulp tissues, pathological changes, lesions or conditions can occur. Often they are caused by physiological factors, such as development, chewing and aging. Furthermore, pathological factors, such as dental caries, food impaction, and mechanical stress injury, can cause pulpal tissue lesions. Injury from mechanical stress may include orthodontic tooth movement and immediate tooth separation during conservative dental treatment. We have already examined the immunohistochemical expression of hard tissue related factors: Runx 2 and alkaline phosphatase (ALP) activities, which may be related to odontoblastactivity up-regulation $^{1,2)}$. Furthermore, we have examined the HSP27 expression in the dental pulp tissues caused by orthodontic mechanical stress ${ }^{3}$. HSPs are proteins acquired for survival under harsh environmental conditions in the cell; they are induced by non-physiological stimuli, as molecular chaperone ${ }^{4)}$ with antiapoptotic features, which allows the cells to escape from death ${ }^{5,6}$. In addition, many cells, in response to stress, inhibit protein denaturation by repairing denatured proteins which express HSPs. We also examined immunohistochemically the expression of HSP27 appearing in mouse dental pulp tissues after experimental immediate tooth separation ${ }^{7}$.

Correspondence to: Dr. Keisuke Nakano, Hard Tissue Pathology Unit, Matsumoto Dental University Graduate School of Oral Medicine, 1780 Hirooka-Gobara, Shiojiri, 399-0781 Japan; Phone \& Fax: +81-263-512035; E-mail: keisuke1@po.mdu.ac.jp
Thus, in the present examination, we examined the expression of HSP70 appearing in the mouse dental pulp after immediate tooth separation, using the same examination model as Saito et al. $(2011)^{7}$.

\section{Experimental animals}

We used in this examination 18 8-week-old ddY mice (weighing $35 \pm 5 \mathrm{~g}$ ), which were purchased from Japan SLC Inc (Hamamatsu, Japan). They were kept in an air-conditioned room with controlled temperature at $24 \pm 1^{\circ} \mathrm{C}$. Animals were reared in paper-lined plastic cages (Paper Clean: Peparlet Co., Ltd, Shizuoka, Japan). Mice were freely fed with solid feed (Picolab Rodent Diet 20: Japan SLC Inc, Hamamatsu, Japan) and water. The ethics committee on laboratory animals at Matsumoto Dental University approved the examination.

\section{Animal experiment}

For the examination, the mice were subjected to inhalation anesthesia with isoflurane (ISOFLU: Dainippon Sumitomo Pharma Co. Osaka, Japan) and gas air mixture (4.0\% preconcentration of anesthesia). The experiments were performed under a stable anesthetic concentration with adjustable constant flow rate of gas anesthesia system for small laboratory animals (DS Pharma Biomedical Co. Ltd, Osaka, Japan). After induction 
J.Hard Tissue Biology Vol. 22(1):7-12, 2013

Table 1. Experimental Periods and Number of Specimens

\begin{tabular}{lccccccc}
\hline & 0-minute & 3-hour & 9-hour & 24-hour & 3-day & 1-week & Total \\
\hline Experimental & 3 & 3 & 3 & 3 & 3 & 3 & 18 \\
Control & 6 & & & & & & \\
\hline
\end{tabular}

of anesthesia, the upper body of the mouse was fixed on a homemade bench. Isoflurane inhalation anesthesia was allowed to flow and maintained during the experiment by inserting a suction hole in the nose from time to time $(1.0 \%$ maintenance concentration). In order to keep the mouth open during the experiment, the jaw was fixed with a thread tied on the upper incisor from the top of the bench and the lower jaw was hung and fixed on the lower bench with a thread tied on the lower incisors. Then a wedge (Anatomical Wizard Wedges: Water Pik, Inc., Colorado, U.S.A.) was inserted in between the right maxillary first (M1) and second (M2) molars. For 3 hours, the wedge was left inserted in between teeth, meaning the separation time was 3 hours. Then the animals were divided into the following groups: 0 minutes, 3 hours, 9 hours, 24 hours, 3 days and 1 week after the removal of the wedge until the mouse was sacrificed for histopathological examination. A total of 6 experimental groups were formed. The number of samples in each group are shown on Table 1. When the duration of anesthesia had lapsed after each experiment, isoflurane inhalation was similarly given. The tissue sample excised consists of a portion of periodontal tissue of the maxillary molars.

For the control group, the left maxillary periodontal tissue of the same subject (opposite untreated side) was used. In this study, the largest tooth closest to the insertion of the wedge would be considered to receive the largest mechanical stress. Although the maxillary $2^{\text {nd }}$ molar is the largest tooth, observation was done on the distal part of the buccal root of the maxillary $1^{\text {st }}$ molar.

\section{Histopathological examination}

The maxillary bone together with maxillary molars and periodontal tissues were removed and immediately fixed in $4 \%$ paraformaldehyde in $0.05 \mathrm{M}$ phosphate buffer for 24 hours. After fixation, the specimens were demineralized in $10 \%$ EDTA solution for 3 weeks. Tissues were embedded in paraffin, and serial horizontal sections of $4 \mu \mathrm{m}$ thickness were cut and subjected to histological and immunohistochemical analyses.

\section{Immunohistochemical examination}

For immunohistochemical staining, the slides were deparaffinized in xylene and pretreated in incubator at $60^{\circ} \mathrm{C}$ for 30 min. Immunohistochemistry was done with Dako Envision + Kit-K4006 (Dako, Glostrup, Denmark). The primary antibody used was rabbit polyclonal HSP70 antibody (HSP70 [K20]: sc-1060,
Santa Cruz Biotechnology, Inc, CA, USA) with a dilution of 1:5000. Specimens were counterstained with hematoxylin. For negative control, PBS was used instead of primary antibody.

\section{Results}

\section{Histopathological examination results}

In control group specimens, maxillary molar pulp tissue was observed as follows. The pulp cavity was partially lined by odontoblasts, some in contact with the dentin and/or some overlapping each other. In some cases, the capillaries were observed within the odontoblast layers. In the central portion of the dental pulp, there were numerous pulp cells and capillaries.

In experimental group specimens, some congestion was generally evident, causing bleeding within the odontoblast layers and central pulp tissues, and containing capillary vessel cells.

\section{Immunohistochemical examination results}

In control group specimens, HSP70 was weakly positive in the cytoplasm of endothelial cells in the odontoblast layers (Figure 1, a). Furthermore, there were some weakly positive staining products within the cytoplasm of dental pulp cells.

The results of experimental group specimens can be described as follows. At first in experimental group 0-minute specimens, meaning immediately after removing the wedge, HSP70 was expressed in most both capillary endothelial cells and dental pulp cells in the pulp tissues (Figure 1, b). In experimental group 3hour specimens, the positive staining products of HSP70 strongly appeared in the cells of odontoblast layers, cytoplasm of capillary endothelial cells in the dental pulp cells, and cytoplasm of dental pulp cells throughout the pulp chamber (Figure 1, c). In experimental group 9-hour specimens, the expression also appeared, the same as in the experimental group 3-hour specimens (Figure 1, d). In experimental group 24-hour specimens, HSP70 expression increased in the odontoblast layers and capillary endothelial cells of resident pulp cells (Figure 1, e). In experimental group 3-day specimens, HSP70 expression in the odontoblast layer could still be observed, but a decrease in expression was evident. In experimental group 1-week specimens, HSP70 further decreased with no difference compared to the control group specimens.

\section{Discussion}

Immunohistochemical analysis of HSP70 expression using mice after immediate tooth separation was performed in this study. 

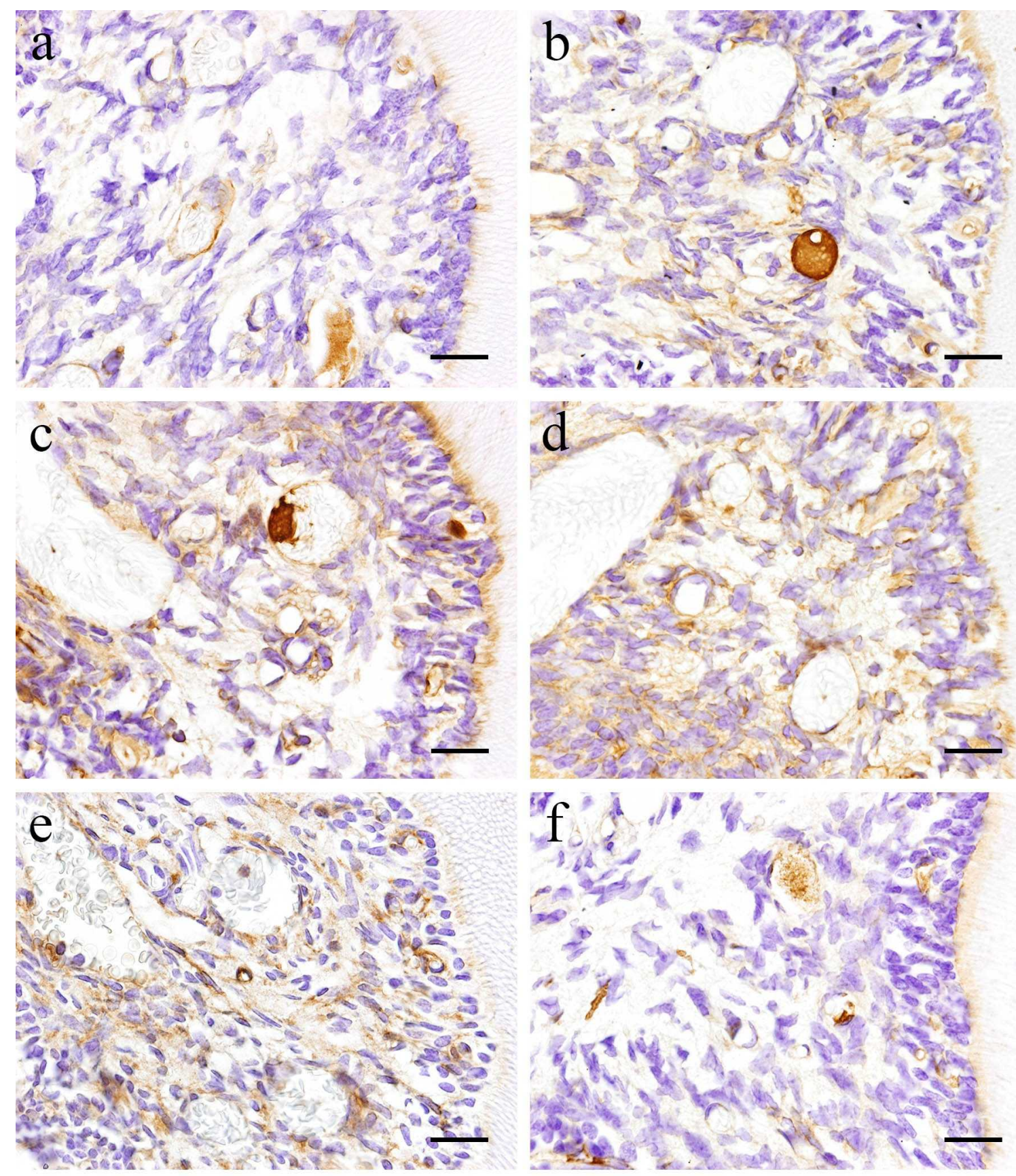

Figure 1. Immunohistochemistry of HSP70 (a: control group specimen; b: experimental 0-min group specimen; c: experimental 3-hour group specimen; d: experimental 9-hour group specimen; e: experimental 24-hour group specimen; and f: experimental 3-day group specimen (Scale bar $20 \mu \mathrm{m}$ ).

Based on our previous report ${ }^{2,7}$, the buccal root of the right maxillary first molar after wedge insertion between M1 and M2 would be the most appropriate area for observation, because it seems to be the most affected region in comparison with the other two roots. We used a 3 -hour wedge insertion period in our previous examinations, and we showed that the results of both the experimental 30-minute group and the experimental 3-hour group were nearly the same. However, the expression was comparatively stronger than that of experimental 30-minute group ${ }^{7)}$.

Regarding the pathological changes occurring in the pulp tissues, there are some examination results that have been published. In general, the pathological changes of the dental pulp tissue occur due to such things as aging, dental caries, occlusal stimuli, traumatic force and orthodontic mechanical stress. ${ }^{8-10)}$. Pathological mechanical stress according to the immediate tooth separation using wedge insertion at the endodontic treatment can 
cause pathological changes in the periodontal tissues. Furthermore, the stress occurs in the pathologically-changed dental pulp tissues, because the stress can also be understood as damage which can act as a stimulus to the dental pulp in the same manner. In other words, the mechanical stress may pass through the nerve and/or vessels relative to the movement in the apical foramen. The stimulus is also thought to take place in the lateral branch. It is for this reason that we planned this present examination.

Regarding the research results of HSPs appearing in dental pulp, some papers have been published ${ }^{11-14}$. We have also examined HSPs in pulp tissues ${ }^{3,7)}$. These examination reports dealt mainly with low molecular weight HSPs, such as HSP 25 and 27. HSP70 is a high molecular weight HSP temporarily attached to the immature state of protein, and known to act as a molecular chaperone with the ability to assist and mature protein folding and to mediate the meeting of polypeptides ${ }^{5,15}$. The speculation that low molecular weight HSPs function as molecular chaperone in cells is not clear $^{4}$.

In our present examination, HSP70 observed in the control group in pulp cells was weak and was mainly expressed by vascular endothelial cells. This expression was noted during the early experimental period, which indicates that HSP70 plays a physiological role in the vascular system ${ }^{5,9}$. In the experimental group, strong HSP70 expression was observed in vascular endothelial cells as well as in some pulp cells. Also, this expression reached its maximum after 24 hours and then gradually decreased until the expression was the same as in the control group. This was due to the stress from separation of teeth, which stimulated some endothelial cells, and their reaction was to express HSP70. This may also be their way to recover from stress. However, the expression was reduced to the same level as that of the control group after one week, suggesting that the injury or the stimulus was not so strong and did not continue very long. Thus, the separation of teeth creates a strong stimulus after about one week as indicated by the expression of HSP70, but the injury is not very destructive to the pulp tissues.

In conclusion, immunohistochemistry was performed to examine the incidence of HSP70 expression. In the experiment group, HSP70 expression increased in the experimental tooth separation group. The expression became the greatest at 24 hours. Then it gradually decreased and became similar to that in the control group at 1 week after the wedge insertion. These results suggest that when immunohistochemical expression of HSP70 is used as an index, there is no severe damage upon clinical application of an inserted wedge.

\section{Acknowledgments}

This study was supported in part by a Grant-in-Aid for Scientific Research (C: 22592303 and 23593075) from the Japan Society for the Promotion of Science. The authors thank Professor
David M Carlson of Matsumoto Dental University for his critical reading of the manuscript.

\section{References}

1. Nakano K, Muraoka R, Tomida M, Matsuura S, Okafuji N, Siar CH and Kawakami T. Possibility of odontoblasts activity up-regulation due to orthodontic mechanical stress in mice. J Hard Tissue Biol 19: 13-16, 2010

2. Nabeyama A, Nakano K, Saito S, Sato M, Okafuji N, Yamamoto A, Kasahara E and Kawakami T. Immunohistochemical expression of hard tissue related factors in the mouse dental pulp after immediate teeth separation. Eur J Med Res 16: 507-513, 2011

3. Nakano K, Muraoka R, Tomida M, Matsuura S, Okafuji N, Siar CH and Kawakami T. Expression of hsp in dental root pulp cells due to experimental orthodontic mechanical stress. J Hard Tissue Biol 18: 127-130, 2009

4. Craig EA, Weissman JS and Horwich AL. Heat shock proteins and molecular chaperones: mediatora of protein conformation and turnover in the cell. Cell 78: 365-372, 1994

5. Schlesinger MJ. Heat shock proteins. J Biol Chem 265: 12111-12114, 1990

6. Gusev NB, Bogatcheva NV and Marston SB. Structure and properties of small heat shock proteins (sHsp) and their interaction with cytoskeleton proteins. Biochemistry (Mosc) 67: 511-519, 2002

7. Saito S, Nakano K, Nabeyama A, Sato M, Okafuji N, Yamamoto A, Kahasara E and Kawakami T. Immunohistochemical expression of heat shock protein 27 in the mouse dental pulp after immediate teeth separation. Eur J Med Res 16: 495-500, 2011

8. Muraoka R, Nakano K, Kurihara S, Yamada K and Kawakami T. Immunohistochemical expression of heat shock proteins in the mouse periodontal tissues due to orthodontic mechanical stress. Eur J Med Res 15: 475-482, 2010

9. Muraoka R, Nakano K, Matsuda H, Tomoda M, Okafuji N, Yamada $\mathrm{K}$ and Kawakami T. A consideration on the role of hsp70 appearing in the periodontal tissues due to experimental orthodontic force. J Hard Tissue Biol 20: 275282, 2011

10. Sato M, Nakano K, Saito S, Nabeyama A, Okafuji N, Yamamoto A, Kasahara E and Kawakami T. Expression of chromogranin A in the mouse dental pulp and periodontal tissues. J Hard Tissue Biol 20: 295-300, 2011

11. Sens DA, McGuirt JP, Khan W, Todd JH and Howell RM. Expression of heat shock protein 27 in adult human third molar dental pulp. J Oral Pathol Med 25: 382-387, 1996

12. Tate Y, Yoshiba K, Yoshiba N, Iwaku M, Okiji T and Ohshima H. Odontoblast responses to GaAlAs laser irradiation in rat molars: an experimental study using heat-shock protein- 25 
Keisuke Nakano et al. : HSP70 in the Dental Pulp after Immediate Teeth Separation

immunohistochemistry. Eur J Oral Sci 114: 50-57, 2006

13. Suzuki T, Nomura S, Maeda T and Ohshima H. An immunocytochemical study of pulpal responses to cavity preparation by laser ablation in rat molars by using antibodies to heat shock protein (HSP)25 and class II MHC antigen. Cell Tissue Res 315: 311-319, 2004

14. Matsuzaka K, Muramatsu T, Katakura A, Ishihara K, Hashimoto S, Yoshinari M, Endo T, Tazaki M, Shintani M, Sato $\mathrm{Y}$ and Inoue T. Changes in the homeostatic mechanism of dental pulp with age: expression of the core-binding factor alpha-1, dentin sialoprotein, vascular endothelial growth factor, and heat shock protein 27 messenger RNAs. J Endod 34: 818-821, 2008

15. Nakakura-Ohshima K, Watanabe J, Kenmotsu S and Ohshima H. Possible role of immunocompetent cells and the expression of heat shock protein-25 in the process of pulpal regeneration after tooth injury in rat molars. J Electron Microsc (Tokyo) 52: 581-591, 2003 
J.Hard Tissue Biology Vol. 22(1):7-12, 2013 